\title{
ANALYSIS OF NANO CHANNEL FORMATION IN QUARTZ CUBES BY LASER-INDUCED PROCESS*
}

\author{
QIN S. J. (秦水介) $)^{1,2,3, \dagger}$ LI Wen J. (李文荣) ${ }^{2}$ \\ ${ }^{1}$ (Laboratory for Photoelectric Technology and Application, Guizhou University, Guiyang 550025, China) \\ ${ }^{2}$ (Center for Micro and Nano Systems, The Chinese University of Hong Kong, Hong Kong SAR, China) \\ ${ }^{3}$ (LNM, Institute of Mechanics, Chinese Academy of Sciences, Beijing 100080, China)
}

\begin{abstract}
A novel laser processing technique was developed for making channels in the nano regime in this paper. A Nd:YAG laser was used to dry fabricate micro channels $(25 \mu \mathrm{m} \sim 100 \mu \mathrm{m}$ diameter) in a $1 \mathrm{~cm}^{3}$ fused silica substrate by thermal-induced processing. By controlling the locations of these initiating micro channels on a silica cube, 1D-controllable self-connecting nano fractures can be formed as rectangular channels. These nano channels are smooth and with extremely high aspect ratio ( $\sim 10^{4}$ depth to width ratio). A possible mechanism is proposed to explain the formation of the nano channels. This laser-based nano channel fabrication technique is fast and inexpensive, and with potential applications in capillary electrophoresis and electro-osmosis driven nano-filtration.
\end{abstract}

KEY WORDS: laser micromachining, nano channel, laser-induced splitting, fracture mechanics

\section{INTRODUCTION}

Nano channels on a quartz, even though it is still being developed at an early stage, will become a promising structure for many fluidic systems such as capillary electrophoresis $(\mathrm{CE})$, which has been refined and is replacing the conventional gel electrophoresis as a more versatile technology in analyzing proteins and DNA's in the past decade ${ }^{[1]}$. Fused silica micro channels (typically $10 \mu \mathrm{m} \sim 100 \mu \mathrm{m}$ diameter) are used as capillaries in CE. By further decreasing the dimensions of the capillary, the negative effects of joule heating in electrophoresis can be reduced since the temperature distribution in the channel is more uniform, and heat is better dissipated ${ }^{[2]}$. The result of this is that more data can be analyzed in shorter time. When a rectangular channel with a high aspect ratio (depth/width) is used, the optical on-column detection sensitivity is improved significantly since the surface to volume ratio is larger. Potentially 2D separation can also be realized in the rectangular channels.

Conventional micro-fabrication methods such as masking and substrate etching have been reported by
Matsumoto et al. in Ref.[3] to make micro-to-nano channels. While highly precise and reliable, the use of e-beam lithography and RIE etching can be expensive and time consuming. Qin and $\mathrm{Li}$ have shown a novel technique in creating complex micro channels using a Nd:YAG laser in a dry process ${ }^{[4,5]}$. This paper reports the use of this technique to create nano fractures as fluidic channels. The formation mechanism of the controllable nano fracture channels is analyzed based on the energy balance theory of the fracture mechanics. Potential applications for these nano-sized rectangular channels include capillary electrophoresis and nano-filtration.

\section{FABRICATION}

A Q-switched Nd:YAG laser, $40 \mathrm{~W}$ of maximum power (Electrox Scriba II D40, UK), was used in our work. The frequency of the laser pulse was set at $2 \mathrm{kHz}$ and the pulse width of the laser was $100 \mathrm{~ns}$ $\sim 300$ ns. The laser beam was focused by a scanning lens ( $\mathrm{f} \cdot \theta$ lens) with a focal length of $100 \mathrm{~mm}$ onto the sample surface. The Gaussian spot size of the beam was determined to be about $400 \mu \mathrm{m}^{2}$ at $1064 \mathrm{~nm}$ (ra-

\footnotetext{
Received 6 August 2003

* The project supported by the National Natural Science Foundation of China (50375031) and the Hong Kong Research Grants Council (CUHK 4416/99E)

† E-mail: sqin@acae.cuhk.edu.hk
} 
dius of $\sim 11.3 \mu \mathrm{m}$ at $1 / \mathrm{e}^{2}$ intensity). All channels were formed in optical grade fused quartz cubes (bubble free synthetic silica, Almaz Optics, Inc., USA). The drilling processes were carried out in air with atmospheric pressure with the laser beam directly focused on the substrates (maskless).

\subsection{Fabrication of Micro Channels}

As reported by $\mathrm{Qin}$ and $\mathrm{Li}^{[4,5]}$, Nd:YAG lasers can be used to create micro channel systems bounded by true solid $3 \mathrm{D}$ quartz substrates without damaging the substrate surfaces by means of the plasma-induced or the thermal-induced method. These techniques surmounted the problem of the quartz being transparent to the spectrum from UV to near IR wavelength by means of the "reverse-cut" process. The thermalinduced process is capable of constructing complex 3D micro channel systems inside quartz substrates and the plasma-induced process can fabricate up to $4 \mathrm{~mm}$ long high quality channels. The channel dimension can be controlled from $25 \mu \mathrm{m}$ to $200 \mu \mathrm{m}$ by setting the dimension of the drilling pattern using a computer controlled laser system.

Photos of micro channels fabricated by the thermal-induced process (at the laser fluence at the rear surface of a quartz substrate about $160 \mathrm{~J} / \mathrm{cm}^{2} \sim 370 \mathrm{~J} / \mathrm{cm}^{2}$ ) are shown in Fig.1. Quartz is a fragile material, the channel shape depends strongly on the state of stresses of the quartz during the laser interaction. As shown in Fig.1(b), there are many thermal cracks around the channel. Also, residual stresses in the quartz may cause secondary fracturing. This effect affects the channel cross-section and reduces the straightness of the channel.

In the plasma-induced process, a quartz substrate was first pre-damaged at a site by the thermalinduced process, and the subsequent laser pulses could be easily absorbed by this locally damaged site. Then the laser beam was focused on this initiated site on

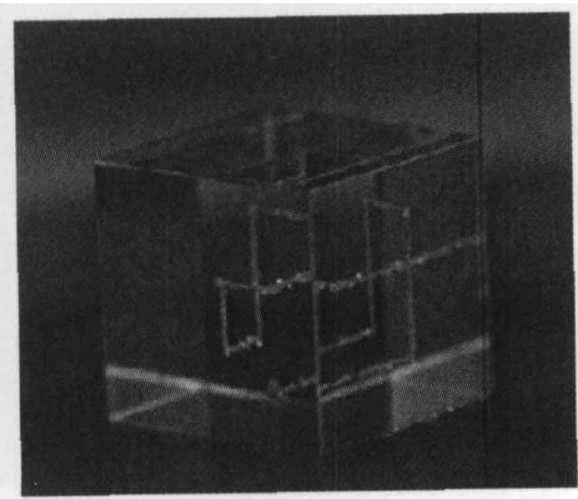

(a) A complex 3D micro channel structure

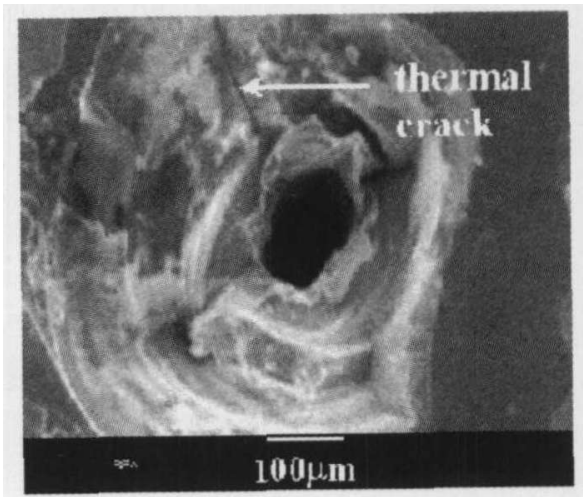

(b) A SEM photo of micro channel

Fig.1 Thermal-induced fabricated channels

the quartz surface. Once the damaged site began to absorb enough laser energy, a hot and dense plasma of quartz was produced around this site. The plasma attacked and melted the neighboring quartz and then a hollow was formed. The hollow functioned as a cavity for the subsequent laser pulses. The laser-induced plasma of quartz was produced in the cavity on each subsequent pulse to drill the hollow further downward, producing a micro channel. The channels fabricated by this process are of high quality with a smooth kerf surface and no thermal cracks are observed, as shown in Fig.2. This process can also be used to smooth-out

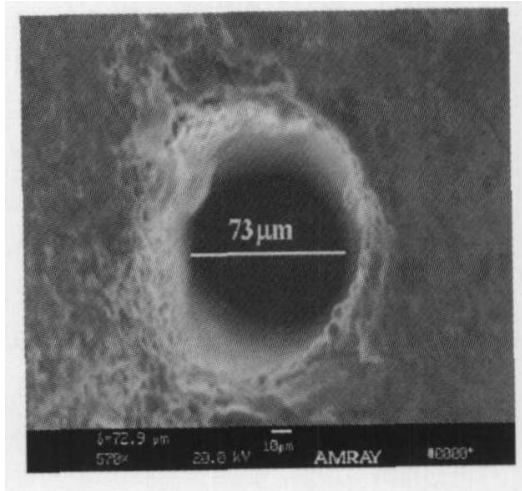

(a)

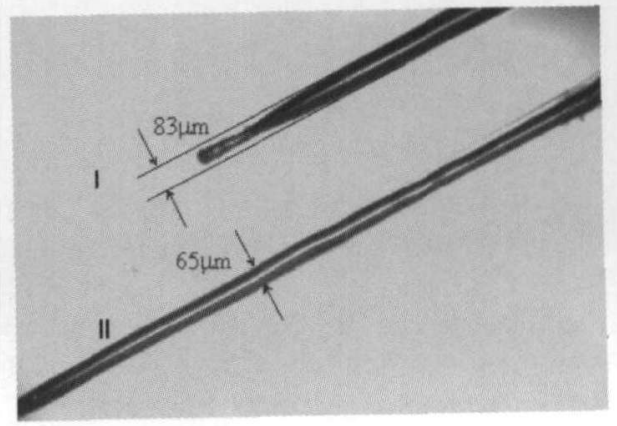

(b)

Fig.2 Plasma-induced laser-fabricated channels 
the thermal-induced channels; hence, complex channel systems with smooth interior wall can be fabricated inside a quartz substrate by the combination of the two processes.

\subsection{Fabrication of Nano Channels}

By drilling thermally-induced initiating micro channels $(60 \mu \mathrm{m} \sim 100 \mu \mathrm{m}$ diameter $)$ in a special sequence and with certain distances as illustrated in Fig.3, a consistent nano "fracture" propagates along the aligned axis to form nano rectangular channels. We have found that three or more initiating micro channels are necessary to induce a nano channel. The distance between the first two initiating channels needs to be $4 \sim 6$ times of that of the 1st and 3rd initiating channels (see Fig.3). The typical distance between the first two initiating channels is about $1.5 \mathrm{~mm} \sim 3.5 \mathrm{~mm}$ and the distance of the 1st and 3rd initiating channels is $0.5 \mathrm{~mm}$. The reason for this phenomenon is most likely due to the induced stress gradient on the quartz substrate caused by laser-processed cavities and will be discussed later. The widths of the induced channels can be as small as $450 \mathrm{~nm}$. Figure 4 shows a SEM photo of a nano channel. These nano channels are in the form of gap structure and can extend from the top through the substrate $(1 \mathrm{~cm})$ to the bottom or to the middle of the substrate, as shown in Fig.5.

By aligning another three or more initiating micro channels in an axis normal to the original nano channel, a new nano channel will intersect the original to form a $2 \mathrm{D}$ system as shown in Fig.6. These channels are formed and controlled by strategic place-

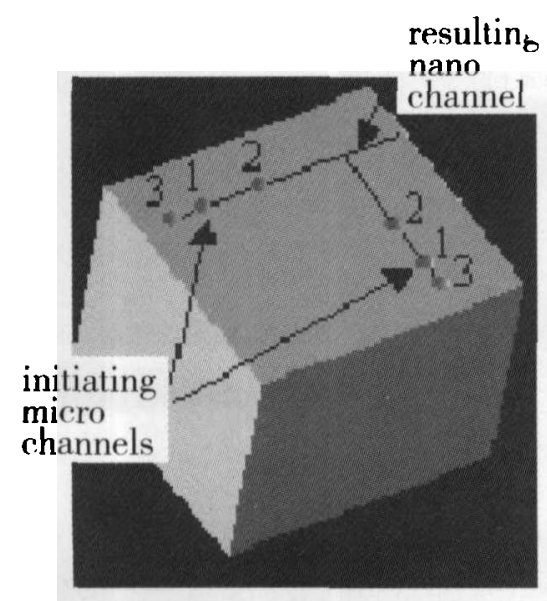

Fig. 3 On a $1 \mathrm{~cm}^{3}$ substrate, the initiating circular micro channels are fabricated in the sequence indicated by the numbers, and the resulting nano channels are shown

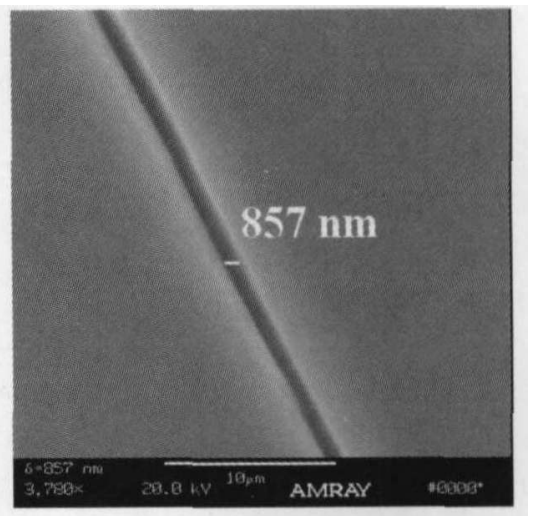

Fig.4 A SEM photo of a nano channels

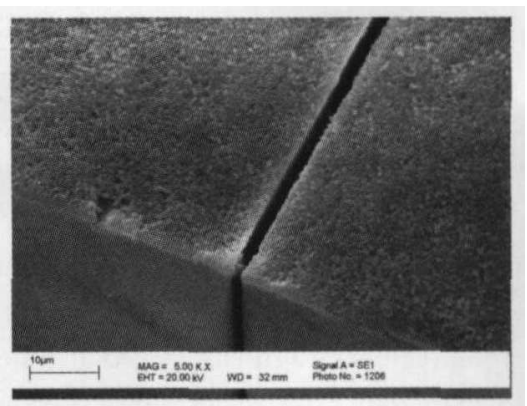

Fig.5 A nano fracture channel is in the form of a gap structure

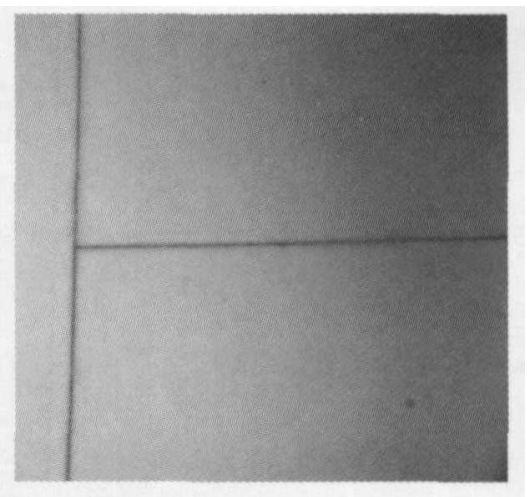

(a)

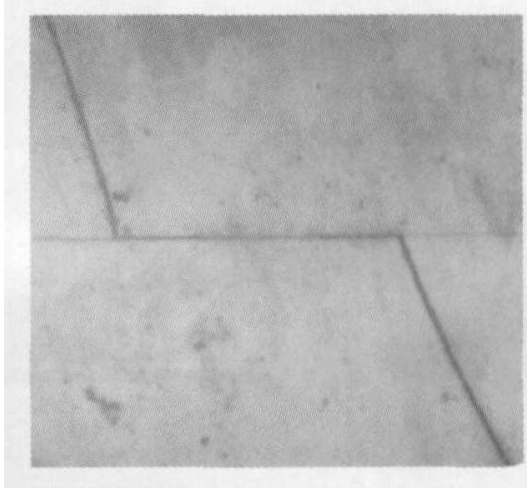

(b)

Fig.6 Microscope photos of nano channels intersecting 
ment of initiating and terminating micro channels. The nano channels can be created in both fused silica and crystalline quartz.

\subsection{Test for Connectivity of the Nano Channels}

Since the nano channels initiate from the micro channels, the micro channels can be used as the sources and drains of cellular samples suspended in a fluid medium and the nano channels can be used as the workspace. We have verified that these interconnected channels are continuous by injecting $\mathrm{FeCl}_{3}$ conductive dye into an inlet micro channel. The channels were proved to be connected visually, as shown in Fig.7. Connectivity was also tested by measuring the resistance between an inlet and an outlet micro channel connected by a nano channel and also a system of connecting nano channels. For the test involving a nano channel between two micro channels the dye was injected at $t=0$ when the nano channel was dry and resistance cannot be measured. At $t=1 \mathrm{~s}$, the dye has propagated through the micro and nano channel to yield a measurable resistance. The resistance decreases until $t=4.5 \mathrm{~s}$ where it begins to increase due to dye depletion as the bulk moves to other interconnecting nano channels. A plot of this resistance measurement over time is shown in Fig.8.

Currently, we are investigating various cellularsample insertion methods to the inlet micro channels. This is in preparation for nano filtration through the nano channels. We have successfully injected saccharomyces cerevisiae cells via capillary transport as well as insertion of the cells by electro-osmosis shown in Fig.9 and Fig.10. Our goal is to perform nano filtration to separate bacteria (nano scale) from cells (micro scale).

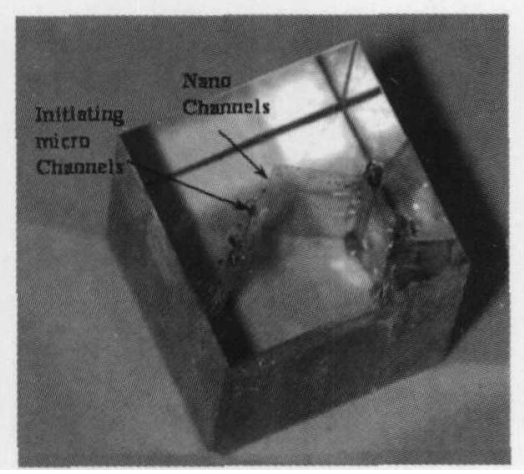

Fig.7 Nano channels tinted with dye. The dye was injected from the micro channels and the capillary force transported it into the nano channels

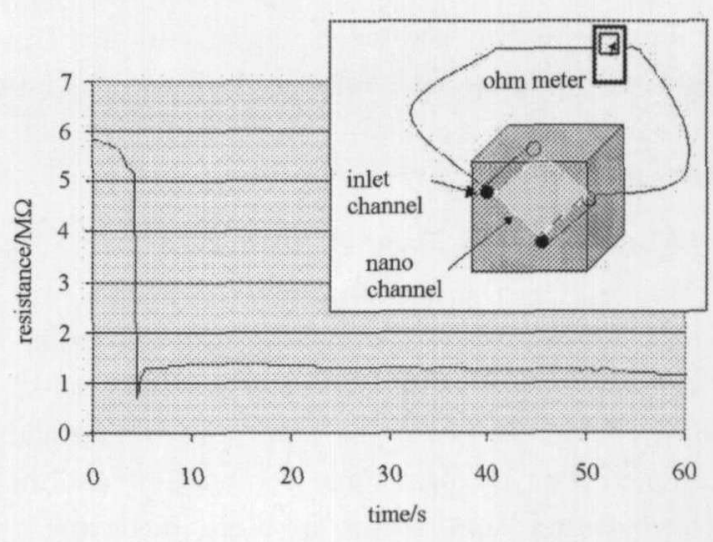

Fig. 8 Test to validate the throughness of a nano channel

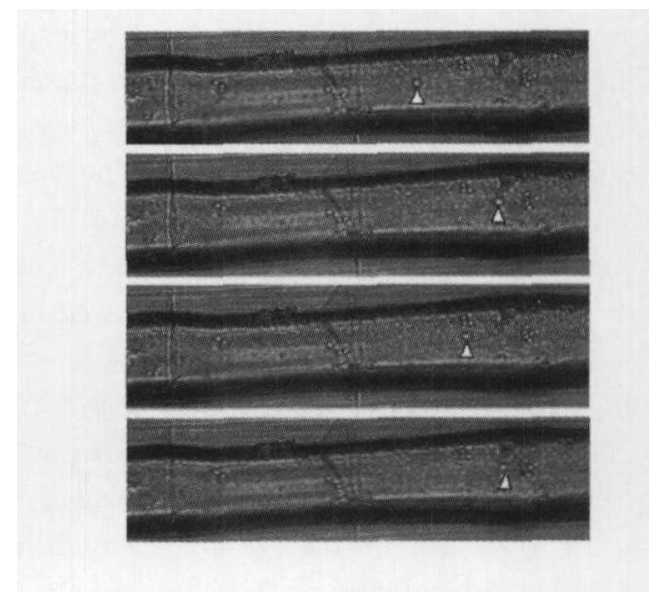

Fig.9 Electro-osmosis driven flow to insert cells into a source micro channel. The arrow follows a cell strand flowing from right to left in electric field of $100 \mathrm{~V} / \mathrm{cm}$ (Cell is color enhanced for easier visualization)

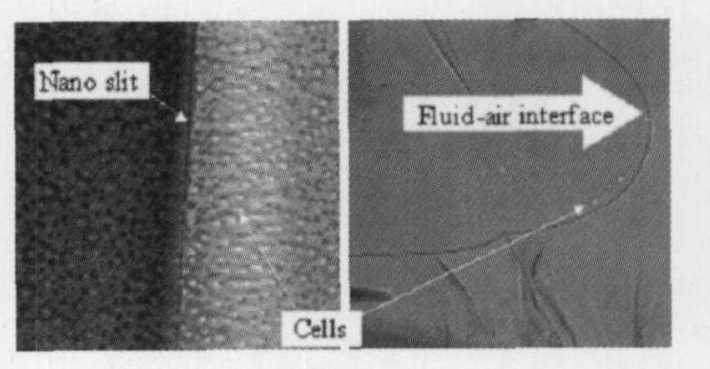

Fig.10 Filtration by a nano channel

\section{DISCUSSION ON NANO CHANNEL FORMATION}

\subsection{Internal Stress Measurement and Function Analysis of Stresses \\ We speculate that these nano fractures are caused by thermally induced residual stresses on the}


bulk quartz substrates during laser-micromachining of the micro channels; this is evident by the stress field measured around an initiating micro channel after laser processing. The stress gradient extends radially and circumferentially. The numeric stress plots of the radial stress, the circumferential stress, and the axial stress, and their directions are shown in Fig.11. From the figure we can see that the circumferential stress, $\sigma_{\theta}$, is positive, and hence is a tensile stress. While the radial stress, $\sigma_{r}$, is a compression stress $\left(\sigma_{r}<0\right)$.

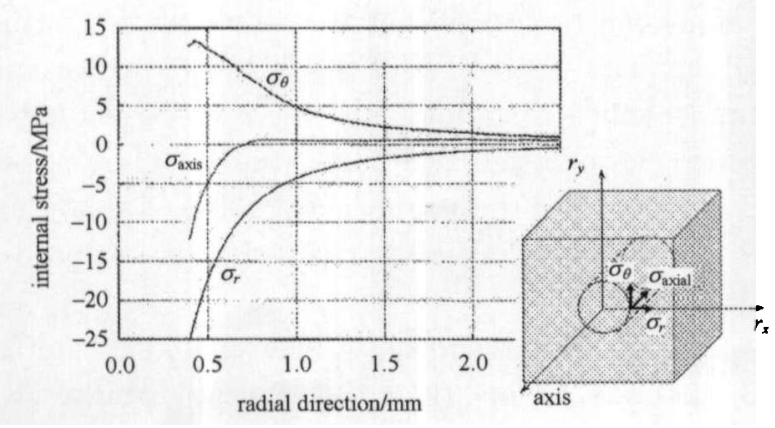

Fig.11 Stress plots on a fused silica after making an initiating micro channel and their directions. The parameters, $\sigma_{r}$, $\sigma_{\theta}$, and $\sigma_{\text {axis }}$ are radial stress, circumferential stress, and axial stress, respectively

In order to understand the effect of stresses on fractures, the energy balance theory of fracture mechanics is introduced to analyze the fracture formation. There are three modes of fracture based on the fracture mechanics: mode I-opening mode, mode II-sliding mode, and mode III_— tearing mode, each of which has its special direction of the strain force caused. For the mode I fractures (opening mode), the direction of the strain force caused is perpendicular to the fracture direction. In our experiments, the nano channels induced appear mainly in the form of mode I fractures (opening mode). The direction of the circumferential stress is perpendicular to a radial direction, which is the direction of a nano channel. It is conjectured that the circumferential stress is possibly the main reason to cause the nano channel, and hence the circumferential stress, $\sigma_{\theta}$, and its effect on the mode I fracture is discussed.

\subsection{Quantitative Analysis of the Circumferen- tial Stress, $\sigma_{\theta}$}

To analyze the stresses more clearly, the circumferential stress plot is re-coordinated from the initiating channel wall to the radial direction axis and is factitiously divided into two parts: the near field when the depth of the channel wall is not more than $0.6 \mathrm{~mm}$, in which the circumferential stress decreases sharply, and the far field when the depth of the channel wall is more than $0.6 \mathrm{~mm}$, in which the circumferential stress decreases gradually, as shown in Fig.12. In the near field, the circumferential stress decreases sharply from the maximum stress about $13.4 \mathrm{MPa}$ at channel wall to $4.9 \mathrm{MPa}$ at about $0.6 \mathrm{~mm}$ of depth, only about one third of the maximum stress. In the far field the circumferential stress decreases very gradually.

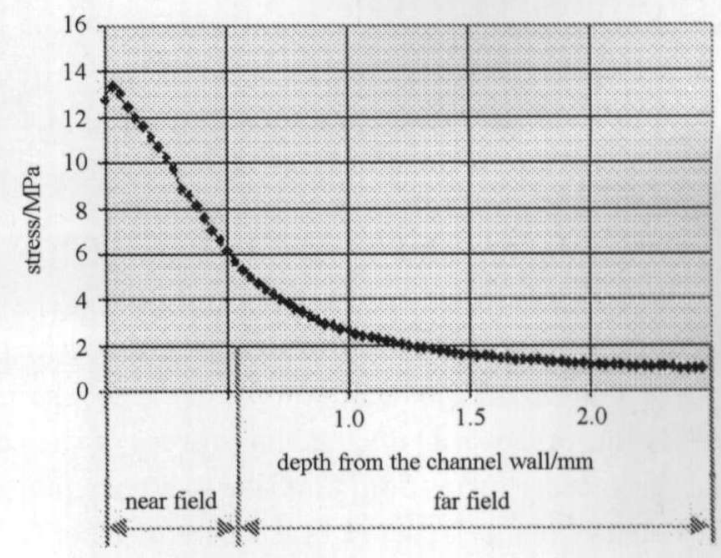

Fig.12 The circumferential stress plot caused by one initiating micro channel

When the second initiating channel is drilling in a typical distance (e.g. $2.5 \mathrm{~mm}$ ) from the first initiating channel, its circumferential stress plot may be made in converse coordinated direction to the first channel, as shown in Fig.13 (plots of channel I and

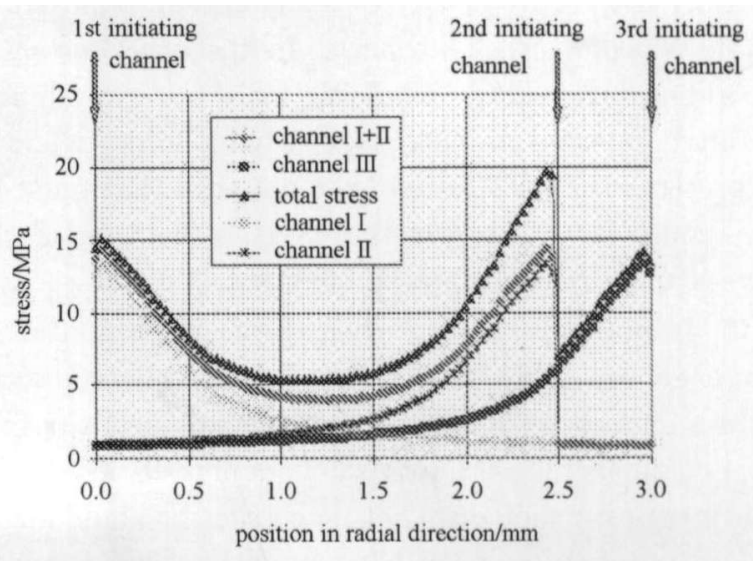

Fig.13 The circumferential stress plots of three initiating channels and the total stress plot vs position. The distances between initiating channels are $2.5 \mathrm{~mm}$ and $0.5 \mathrm{~mm}$, respectively 
channel II), and the total stress plot of the first two channels is got by superposition (see Fig.13, plot channel I+II). We may guess that there will be a basic uniform stress field between the two channels, in which the stress is less than the threshold of fracturing. The uniform field is located in the far field of both initiating channels. When the third initiating channel is aligned on the axis of the first two initiating channels and the distance to the 1st (or 2nd) channel is less than $1 \mathrm{~mm}$ (typical $0.5 \mathrm{~mm}$ ), the total stress of the far field is then significant high enough to cause a large crack firstly in the area between the first two channels (see Fig.13, plot total stress). The crack is then propagates along the aligned axis outward the 2nd (or 1st) channel to form a long and straight fracture.

\subsection{Fracture Formation Analysis by Energy Balance Theory}

It is supposed that the energy remained in the far field before drilling the third initiating channel plays an important role during the process of the controllable fracture formation, and this will be explained by the energy balance theory of fracture mechanics. It is known from the theory that when a solid substrate is fractured, new surfaces are created in the medium in a thermodynamically irreversible manner. Material separation is caused by the rupture of atomic bonds due to high local stress. If the strain energy released when a crack grows a small amount is equal to or larger than the energy required to form the new crack surfaces, then the crack will continue to grow. The condition for growth can be written symbolically as $G \geq S$, where $G$ is the strain energy release rate per unit of crack growth and $S$ is the energy required to form two new crack surfaces. In the other word, $G$ represents the energy available for crack growth and is often referred as the crack driving force, while $S$ represents the resistance of the material that must be overcome for crack growth. $G$ is a function of the stress while $S$ is a material constant.

In our experiment, when the first two initiating channels are drilled in a right distance, there is some strain energy accumulated in the far field of the first two channels before the third channel is drilled due to the stresses, which does not reach the threshold stress $\sigma_{\text {th }}$ to induce a crack. When some new stress is added to the original stressed field by drilling the third initiating channel and thus the total stress reaches the threshold stress $\sigma_{\text {th }}$, a new crack is created. At the same time, the original stored energy will be released to grow the crack forward. The power of the original stored energy may be much higher than that of new added energy since it can grow the crack through the 1st (or 2nd) initiating channel outward much longer than cracks around any of initiating channels due to stresses.

The hypothesis that the nano fracture is possibly induced by the energy remained in the far field is verified by the following experiments. When the distance of the first two initiating channels is larger than the typical distance (e.g., $\sim 4 \mathrm{~mm}$ ), the long straight crack cannot grow through any outboard initiating channels to form a long and straight crack, i.e., only a short channel can be formed between the first two initiating channels. Also, even if the 3rd, 4th, or 5th initiating channels are added, a straight crack can not be formed through the initiating channels. This is possibly because that the strain energy released is not high enough when the initiating channels are separated at that distance.

When the distance of the first two initiating channels is less than the typical distance (e.g. $\sim 1 \mathrm{~mm}$ ), the crack cannot be propagated outward either, no matter how the third initiating channel is drilled. The reason is possibly that the uniform stress field like in the far field is not formed at this distance. Figure 14 shows the circumferential stress plots of two initiating micro channels with the distance of $1.0 \mathrm{~mm}$. In this distance, the near field of two channels is overlaped and the total stress between the two channels is very high. It will cause many irregular and unexpected cracks around the initiating channels and thus the strain energy is released completely at the same time.

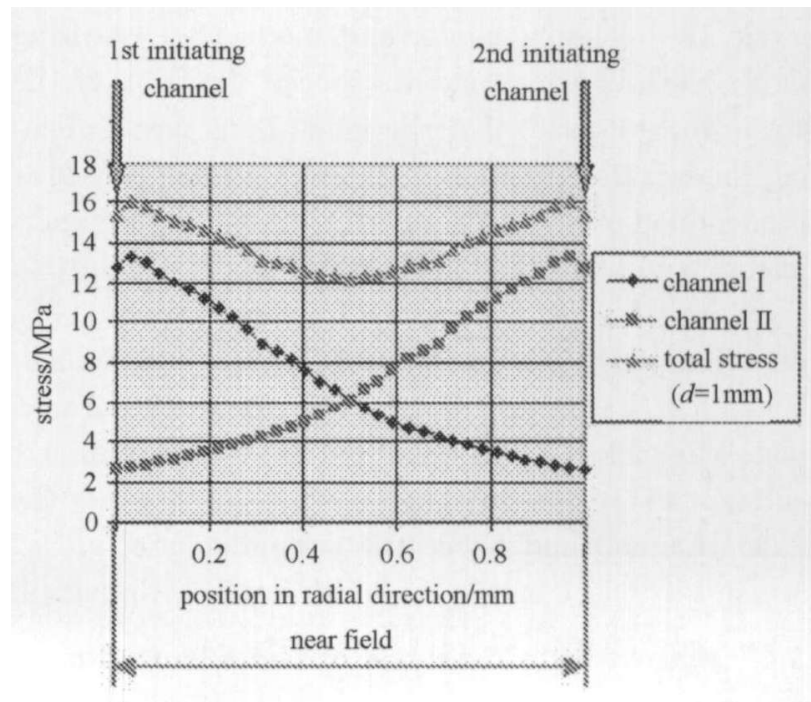

Fig.14 The circumferential stress plots of two initiating micro channels with the distance of $1.0 \mathrm{~mm}$ 
More detailed experimental tests on determining the nano channel geometries with the locations of the micro channels are underway in our group. These stress fields do not exist when there are no micro channels in the quartz substrate.

\section{CONCLUSION}

We have developed a novel laser processing technique for making channels in the nano regime using a Nd:YAG laser in this paper. By controlling the locations of the initiating micro channels on quartz substrates, 1D-controllable self-connecting nano fractures can be formed as rectangular channels. These nano channels are smooth with extremely high aspect ratio. We speculate that these nano fractures are possibly induced by the energy remained in the far field of the first two initiating channels. This technique will be important for the advancement of making many fluidic systems useful to improve performance and add new functionality to many applications such as chemical, blood, DNA, and environment science analyses. Applications include studies of cell-to-cell interaction and cell culturing where nano-holes can be used to filter out virus and damaging particulates and allow nutrients to get to the cell, thus allowing the cell to live for a very long time. Future work involves fluid flow visualization of the nano channels, cell culturing, DNA and molecular manipulation, and cell-to-cell interaction studies in the nano channels.

Acknowledgements We would like to thank the Laboratory of Photoelasticity, Institute of Cybernetic, TU Tallinn, Estonia, for providing the quantitative analysis of the stress distribution around the micro channels.

\section{REFERENCES}

1 Chapman J, Hobbs J. Putting capillary electrophoresis to work. LC-GC Magazine, 1999, 17(3): 86 99

2 Kuhn R, Hoffstetter-Kuhn S. Capillary Electrophoresis: Principles and Practice, Berlin: Springer-Verlag, 1993

3 Matsumoto K, Kawakamiv T, Nakao M, et al. Nanochannel on quartz-chip laboratory using single molecular detectable thermal lens microscope. In: IEEE MEMS, 1998. 127 130

4 Qin SJ, Li WJ. Fabrication of complex micro channel systems inside optically-transparent 3D substrates by laser processing. In: 11th Inter Conf on Solid-State Sensors and Actuators (Transducers '01/Eurosensors $\mathrm{XV}), 2001.1624 \sim 1627$

5 Qin SJ, Li WJ. Micromachining of complex channel systems in 3D quartz substrates using Q-switched Nd: YAG laser. Applied Physics A, 2002, 74(6): 773 777 